\title{
Online Teaching Evaluation System Design and Implementation
}

\author{
Baosheng Qi \\ Department of Information Engineering and Art Design, Shandong Vocational \\ College of Labor, Jinan, 250022, China
}

\begin{abstract}
Student evaluation of teaching is a student to teacher's teaching work evaluation, to carry out this work is the starting point of improving teachers' teaching quality. This paper discusses the use of ASP.NET and SQL Server technology design and implementation of an online teaching evaluation system. The whole system is divided into students operating and backstage management of two modules, the system needs to design the database of the evaluation of teachers' work, focusing on the Session object, data aggregation processing, validation, and other key technologies, and finally introduce the entire system to run the application instance and effect, and shows the main functions of the system. Compared with other similar systems, it does not usually need to have large amounts of data management and maintenance.
\end{abstract}

Keywords:teaching evaluation system, ASP.NET, SQL Server, ADO.NET, session validation

\section{Introduction}

With the deepening and development of higher education reform in our country, more and more people pay attention to the quality of teaching. The teaching quality evaluation has become an inevitable trend. Students' evaluation of teaching is the evaluation of teachers' teaching work. The starting point of this work is to improve teachers' teaching quality. In China's universities, the more standardized student evaluation of teaching activities should be accompanied by the rise of science and higher education assessment activities and gradually formed a good development. The teaching process used by students to fill out a form, the manual summary statistics to traditional students' evaluation of the 
resulting workload, loss, poor timeliness of the drawbacks. Aiming at how to use Web technology to solve the practical problems encountered in the evaluation of university teaching, it is necessary to use computer network, ASP.NET, SQL Server technology to achieve online teaching evaluation system in order to improve the efficiency of teaching evaluation. Based on the actual situation of Shandong Career Academy, this paper designs and implements a simple and easy to use online teaching evaluation system, which plays an important role in the teaching management of colleges and universities.

The class online teaching evaluation system design calculation based on LAN environment of the school, in the class as a unit, organized by teaching evaluation, teaching evaluation method to improve other computer system maintenance, does not need a lot of teachers and students of information usually, the field with a small quantity of data, the system should establish friendly interface. The system interface not only has the advantages of simple operation, bus also should be intuitive, flexible and easy to use.

\section{System design principles}

ASP.NET is a dynamic web page design technology launched by Microsoft Corp. The development speed is very fast. Because the ASP.NET is based on the common language compiler to run the program, so it can run on almost all the Web applicationplatforms. The ASP.NET program uses Visual Studio.NET development environment, WYSIWYG (What You See Is What You Get) editor; ASP.NET is generally divided into two kinds of development language: VB.NET and $\mathrm{CH}$. The $\mathrm{CH}$ is relatively common.

ASP.NET is a Web platform that provides all the services necessary to build a Web application based on enterprise server. ASP.NET is built on the basis of.NET Framework, so all.NET Framework features are suitable for ASP.NET applications.

Teaching evaluation system model makes the system more clear, more clear division of labor, maintenance and upgrade to the system. The user presentation layer provides the client with access to the application, which is implemented by the WEB page. The data access layer is built on the database. The data access layer general package database selection, add, update and delete operations, but also for the presentation layer provides access to the database interface and function; the database layer is at the bottom of the system, all its data storage system, providing access to data in the form of data access layer. In order to achieve the purpose of flexible management of the data on the server side, the three layers of data are transmitted sequentially.

With the rapid development of information technology, database technology, as the core technology of data management, plays an important role in all fields of society. Microsoft released by the SQL Server product is a typical relational database management system, with its powerful features to be recognized by the majority of users. SQL Server 2008 is a relational database management system of the latest release of Microsoft. It provides a reliable, efficient and intelligent 
datacan run the most demanding needs, to complete the mission critical applications.

The SQL Server system consists of 4 parts: the database engine, Analysis Services, Reporting Services and Integration Services. Teaching evaluation only to the database engine service system, the database engine is the core service of Microsoft SQL Server 2008 system, memory and processing relationshiptypes of data or XML data service, responsible for storage, processing and data management and security. Typically, the use of a database system is actually using the database engine.

\section{System design}

\subsection{Main functions of system modules}

The teaching evaluation system is based on NET Framework ASP.NET server script programming environment. It takes Visual Studio as the development platform, the database system uses SQL Server, and the development language uses $\mathrm{C}$ \#. This project is the development environment of Microsoft Visual Studio.NET is a complete set of development tools, used to generate ASP.NET Web applications, which use the.NET Framework function. This framework provides key technology to simplify the ASP.NET Web application development support.

The system uses browser/server (B/S) mode, that is, Browser/Server, compared to $\mathrm{C} / \mathrm{S}$ mode, $\mathrm{B} / \mathrm{S}$ mode does not need to install the client software. $\mathrm{B} / \mathrm{S}$ based on the Intranet principle in the end of all the client side of the maintenance work, also known as "thin client".

The whole system is divided into students' operation and background management of two modules, student operation module is simple and easy to use, complete the simulation process the actual evaluation of the teacher, the whole evaluation process simply enter my student ID once, on evaluation matters individual choices.

In the background management module, the management operations were completed in a page, improve operating efficiency, intuitive interface, add the login page and improve the data security operation, only the correct user name and password to login, complete the corresponding management tasks, including clear evaluation of teaching records.

\subsection{Design of database}

According to the analysis of the main function of the system, we finally determine the data table of the system, and the fields included in each table.The relationship can be observed in Figure 1. It contains four tables, which are teacher evaluation results in table teacher, table result, table inspect, counsellor teacher evaluation table inspect. Compared with the inspectl table inspect table information fixed, it is independent of the table used to store the teacher and counsellor evaluation standard, teacher and counsellor evaluation standard is not 
the same. In the design of the system to delete the student table, the reason is that students are too large quantity of table information, the data is dynamic, the usual management and maintenance workload is very large, the role of information in the system is not the main; in addition, the student table delete, teaching evaluation system can be separated from the whole educational system and increase the flexibility of the system, the system does not require maintenance and daily management. Each semester a general class in $6 \sim 8$ between the door, in the teacher table data in the class teaching evaluation temporary input $6 \sim 8$ lines, each line of input teaName, course two can be listed, and other columns in the result table data or by the student input (ID column stuNo), or by the system automatically generation.

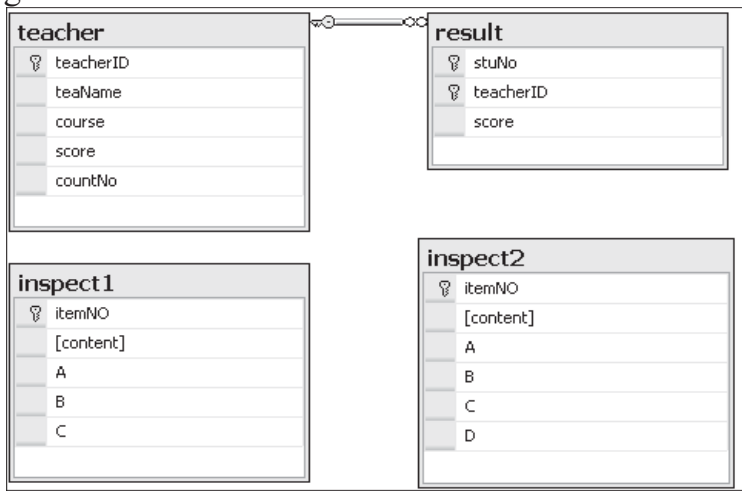

Fig. 1: Relationship figure in the database

\section{Key technologies of system implementation}

\subsection{Passing value between by Session object}

ASP.NET in the realization of the number of pages between the transfer of several methods, the system uses Session objects in the transfer of values between pages. When a student or an administrator when the system is used, the school or the administrator account can be recorded, when users jump in different pages, Session values are not lost, so as to ensure the integrity of the user session. More importantly, at a time when the user opens the page or perform an operation, the system will re verify the Session variable values, thus avoiding the user access across the page resulted in the illegal operation, improve the security of the system.

\subsection{Avoiding repeated evaluations}

To avoid a number of student evaluation of a teacher, the system has adopted two measures: one is in the evaluation of the page set Response.Buffer = true; Response.ExpiresAbsolute $=$ DateTime.Now; Response for the object from the server to send the user to the output, the Buffer property is set to true when the output buffer page, all server script processing after the server only the current 
page will send a response to the client, the ExpiresAbsolute property is set to DateTime.Now immediately expired in the client display page, so that students in the evaluation of data can be submitted through the browser's "back" button to return to the evaluation of the page, to avoid evaluation of the same teacher; the two is to execute the query string strSql = "select count in the database $\left(^{*}\right)$ from result where stuNo $="$ " + stuno + "an $\mathrm{D}$ teacherID $=++$ teacherID; if the query returns a value of 1 , indicating that the student has been evaluated by the teacher, display the appropriate information and jump to the initial page.

\subsection{Distinguishing teachers and counsellors}

The teacher and counsellor information are stored in table teacher, because in the different nature of the work, the teacher and counsellor evaluation standard is not the same, the teacher as the curriculum, counsellors is not teaching, course field in the teacher table that served as the course for teachers, counsellors, this field fill in the value of counsellors, programming according to the value of the course field will be the teachers and counsellors to distinguish, control the page Jump to the teacher or counsellor evaluation page. The advantage of this is to save the cost of the table, convenient programming control.

\subsection{Validation of regular expression}

Class is the basic unit of all kinds of school management, the school often organize the activities of the class as a unit. Our college will be divided into a class A, B two small classes to manage, facilitate Vocational Colleges in two small rotation theory practice class and improve the management efficiency. In the design of the corresponding evaluation consider the teaching system, students enter the school to avoid mistakes, introduces the data validation mechanism in ASP.NET, RegularExpressionValidator controls to limit the input number used in $\mathrm{A} 00 \sim \mathrm{A} 29, \mathrm{~B} 00 \sim \mathrm{B} 29$, the ValidationExpression property is set to [AaBb], so that the evaluation of each student in a certain extent only; if the number is not in the above range, it can appear "please enter the correct number form!" Information, and cannot be verified through the page. The evaluation work cannot be carried out.

\section{Application example and results}

The system needs to be installed on the Windows Server environment, in order to reduce the workload of each department is installed, it can be installed to the college website server, through the links on the web page access, in the nonevaluation time can turn it off, when needed can be opened. To carry out student evaluation of teaching work, as long as the LAN environment, through the IE browser to visit the school website evaluation link. We click the "start to teach" button, enter the evaluation teacher page. The evaluation of the instructor's page is similar to the evaluation of the teacher's page. 


\section{Conclusion}

The subject of the "online Teaching Evaluation System " is aimed at a variety of colleges, schools, primary and secondary occupation educational administration work, whichcan greatly improve the efficiency of educational administrationto improve the accuracy of evaluation. It not only can be used directly in our school, but also has very high popularization value.

\section{References}

[1]Wei Min, A Research on Statistical Information Applied to Tourist Traffic and Transport System Design Based on ASP .NET, Journal of Convergence Information Technology, 6(1), pp. 147-156, 2011.

[2]Chen C.C., Shaw R.S., Knight M.B., THE EFFICACY OF KNOWLEDGE MAP IN OBJECT-ORIENTED SOFTWARE TRAINING: ADO .NET COMPONENTS.MANAGEMENT RESEARCH AND PRACTICE,2(2), pp. 132-153, 2010.

[3]Araby Greene, Managing subject guides with SQL Server and ASP .NET, Library Hi Tech, 26(2), pp. 213-231, 2008.

[4]Ghassan Z.Qadah, Rani Taha, Electronic voting systems: Requirements, design, and implementation, Computer Standards \& Interfaces, (29), pp. 376-386, 2007. 